\title{
Computational Approaches for Pharmacovigilance Signal Detection: Toward Integrated and Semantically-Enriched Frameworks
}

\author{
Vassilis G. Koutkias • Marie-Christine Jaulent
}

Published online: 8 March 2015

(C) The Author(s) 2015. This article is published with open access at Springerlink.com

\begin{abstract}
Computational signal detection constitutes a key element of postmarketing drug monitoring and surveillance. Diverse data sources are considered within the 'search space' of pharmacovigilance scientists, and respective data analysis methods are employed, all with their qualities and shortcomings, towards more timely and accurate signal detection. Recent systematic comparative studies highlighted not only event-based and data-source-based differential performance across methods but also their complementarity. These findings reinforce the arguments for exploiting all possible information sources for drug safety and the parallel use of multiple signal detection methods. Combinatorial signal detection has been pursued in few studies up to now, employing a rather limited number of methods and data sources but illustrating well-promising outcomes. However, the large-scale realization of this approach requires systematic frameworks to address the challenges of the concurrent analysis setting. In this paper, we argue that semantic technologies provide the means to address some of these challenges, and we particularly highlight their contribution in (a) annotating data sources and analysis methods with quality attributes to facilitate their selection given the analysis scope; (b) consistently defining study parameters such
\end{abstract}

V. G. Koutkias $(\bowtie) \cdot$ M.-C. Jaulent

INSERM, U1142, LIMICS, Campus des Cordeliers, 15 rue de l' École de Médecine, 75006 Paris, France e-mail: vasileios.koutkias@inserm.fr

V. G. Koutkias · M.-C. Jaulent

Sorbonne Universités, UPMC Univ Paris 06,

UMR_S 1142, LIMICS, 75006 Paris, France

V. G. Koutkias - M.-C. Jaulent

Université Paris 13, Sorbonne Paris Cité,

UMR_S 1142, LIMICS, 93430 Villetaneuse, France as health outcomes and drugs of interest, and providing guidance for study setup; (c) expressing analysis outcomes in a common format enabling data sharing and systematic comparisons; and (d) assessing/supporting the novelty of the aggregated outcomes through access to reference knowledge sources related to drug safety. A semantically-enriched framework can facilitate seamless access and use of different data sources and computational methods in an integrated fashion, bringing a new perspective for large-scale, knowledge-intensive signal detection.

\section{Key Points}

A number of comparative studies assessing various signal detection methods applied to diverse types of data have highlighted the need for combinatorialintegrated approaches.

Large-scale integrated signal detection requires systematic frameworks in order to address the challenges posed within the underlying concurrent analysis setting.

Semantic technologies and tools may provide the means to address the challenges posed in integrated signal detection, and establish the basis for knowledge-intensive signal detection.

\section{Introduction}

One of the most important aspects of marketed-drug safety monitoring is the identification and analysis of new, 
medically important findings (so-called 'signals') that might influence the use of a medicine [1]. According to the CIOMS VIII Working Group, a signal constitutes "information that arises from one or multiple sources (including observations and experiments), which suggests a new potentially causal association, or a new aspect of a known association, between an intervention and an event or set of related events, either adverse or beneficial, that is judged to be of sufficient likelihood to justify verificatory action" [2].

Computational analysis methods constitute an important tool for signal detection [3, 4]. Lately, the field of signal detection has been very active, with various large-scale collaborative initiatives and projects, such as EU-ADR (http://euadr-project.org/), Mini-Sentinel (http://www. mini-sentinel.org/), OMOP (http://omop.org/), and PROTECT (http://www.imi-protect.eu/). While various advances have been illustrated, e.g. common data models [5], reference datasets for evaluation [6], as well as new analysis methods and systematic empirical assessments [712], the challenge of accurate, timely and evidence-based signal detection still remains [13].

In this paper, we first present a brief overview of postmarketing data sources and computational analysis methods, and highlight their strengths and limitations for signal detection, taking into account recent comparative studies. Under this perspective, we indicate the need for combinatorial signal detection, relying on the concurrent exploitation of diverse data sources and detection methods, and refer to early successful paradigms. We argue that in order to explore combinatorial signal detection in its full potential, semantically-enriched detection frameworks are required to overcome existing barriers. We also illustrate how such a framework can be incorporated within the signal detection workflow, refer to example applications of semantic technologies in drug safety and, finally, discuss this perspective in the scope of large-scale, knowledgeintensive signal detection.

\section{Data Sources and Signal Detection Methods: The Need for Combinatorial Exploitation}

The types of data sources employed for signal detection vary [4]. According to the computational methods adopted/ required for their analysis, we may discriminate the main sources into the following:

1. Spontaneous reporting systems (SRSs) These constitute the dominant signal source through which cases of suspected adverse drug reactions (ADRs) are reported by healthcare professionals or citizens to regulatory authorities or other bodies. Typically, methods for the analysis of SRS data rely on the statistical investigation of disproportionality (DP) [14], or are based on multivariate modeling [3, 4]. A comprehensive review of SRS-based signal detection methods has been presented by Hauben and Bate [15]. Despite SRSs having been quite extensively analyzed, advances on detection methods are still being demonstrated, such as the vigiRank algorithm [16], which combines multiple strength-of-evidence prediction indicators to improve accuracy compared with DP analysis alone.

2. Structured longitudinal observational healthcare databases These are primarily obtained from Electronic Health Record (EHR) and administrative claim systems, and offer the potential to enable active and real-time surveillance [5]. Signal detection methods applied to this type of data typically involve datamining techniques that have their origin from statistical epidemiology [7, 17], e.g. case-control methods [8], cohort methods [11], self-controlled case-series methods [10], and self-controlled cohort design methods [7, 9]. Notably, DP-based methods, originally proposed for the analysis of SRS data, have also been applied to observational data [12], following appropriate extensions and data transformations [18]. A comprehensive review of signal detection methods exploiting observational data has been presented by Suling and Pigeot [19].

3. Unstructured/free-text sources Typical examples include clinical narratives, scientific literature and patient-generated content, e.g. in social media. Extraction of information associating drugs with adverse events from unstructured text requires the employment of text-mining techniques [20]. Clinical narratives are a major part of many clinical information systems and, despite the complexity and barriers in processing clinical text [21], successful information extraction paradigms have been illustrated [22, 23]. The literature has also been explored to provide indications for signals, e.g. by using corpuses extracted from PubMed, and methods relying on the Medical Subject Headings (MeSH) indexing system and statistical inference [24, 25]. Patient-generated data, either shared among networked communities using social media (e.g. blogs, messaging/micro-blogging platforms and forums) [26] or implicitly captured through Web search logs [27], have been more recently explored for signal detection, with interesting findings. A review on text mining for adverse drug event detection considering various types of free-text data has been presented by Harpaz et al. [28].

Each one of the above sources is attributed with advantages and limitations that affect the signal detection 


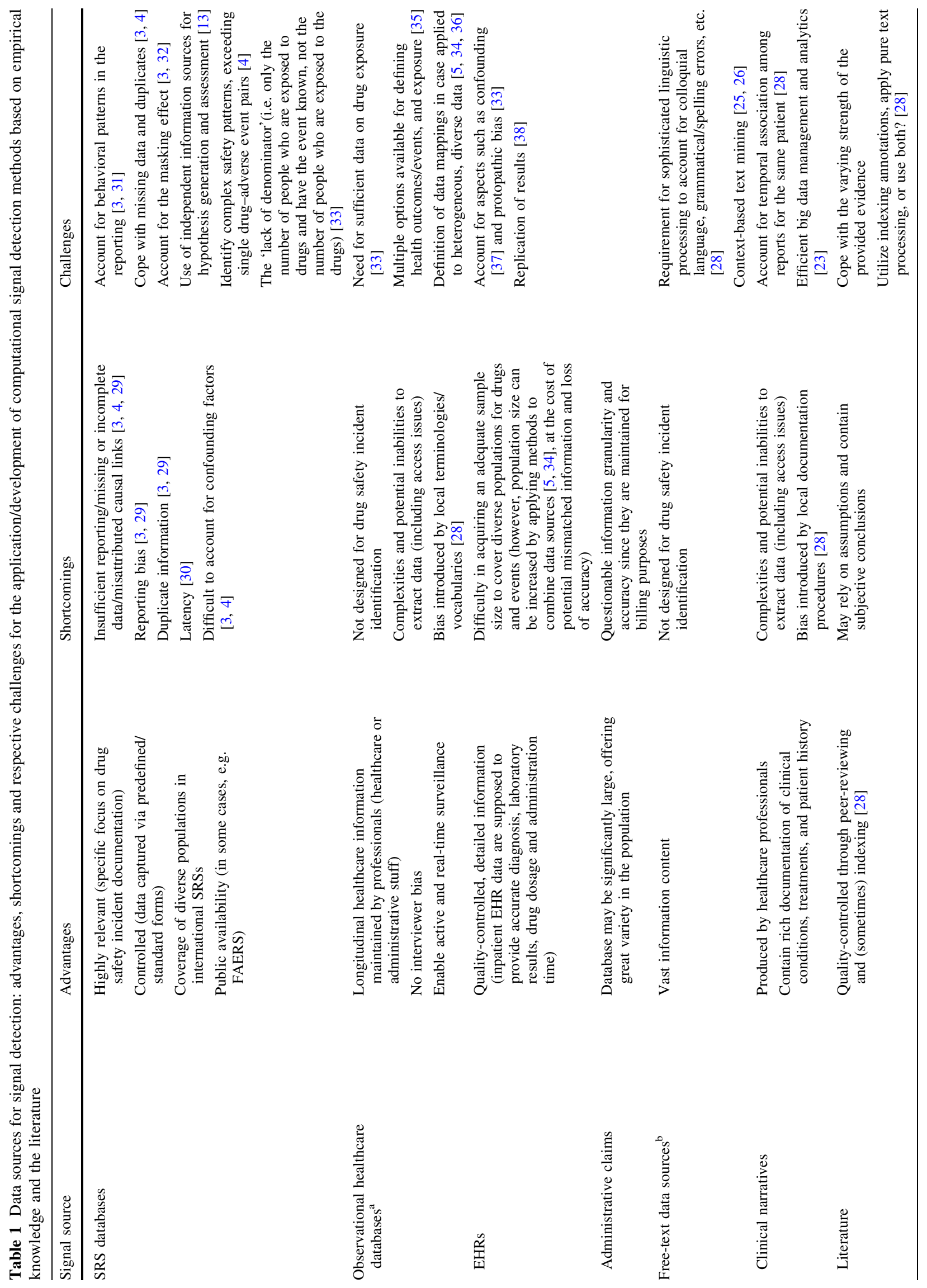




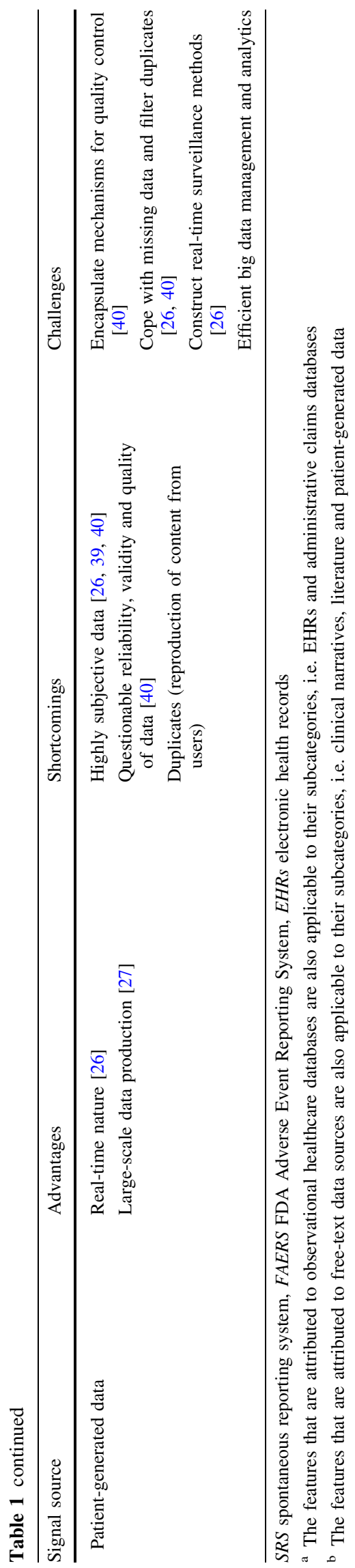

capacity, such as relevance, coverage, quality, reliability and bias, to name a few. Without aiming to cite an exhaustive list, Table 1 summarizes some of these attributes, as well as the challenges involved in using these sources for signal detection based on empirical knowledge and the literature.

In addition, a number of recent comparative studies of signal detection methods exploiting SRS and observational healthcare data illustrated the following (information regarding the employed data, methods and comparison measures, as well as noteworthy analysis choices are summarized in Table 2):

1. Shortcomings in detection accuracy/efficiency A high rate of false-positive indications [38, 43] and difficulties in detecting rare ADRs [43], while some events were not detectable despite the variety of the employed methods [33].

2. Performance variation Event-based differential performance of methods [31], as well as differential performance with respect to the data used for analysis [43, 44].

3. Complementarity A time-to-onset (TTO)-based method with a DP-based method when applied to SRS data [41], and DP-based methods with multivariate-based signal detection strategies exploiting observational data [31], were found to be complementary.

Based on the above remarks, we can conclude that all the available data sources and the concurrent use of different signal detection methods need to be considered in the construction of a holistic signal detection framework. In the following section, we refer to two characteristic studies, which elaborated on combining information across diverse data sources and signal detection methods.

\section{On Combinatorial Computational Signal Detection: Examples}

\subsection{Joint Signaling in a Spontaneous Reporting System and an Electronic Health Record System}

Given the maturity of drug surveillance based on SRS data, the progress made in the use of observational healthcare data, and the expectation that the two sources may complement each other, Harpaz et al. [45] argued that it makes sense to consider computational approaches that may combine information from these two types of sources. The motivation for the study was the assumption that a combinatorial investigation would either lead to increased evidence or statistical power of findings, or would facilitate new discoveries that may not be possible with either source separately. In particular, the study elaborated on the joint 


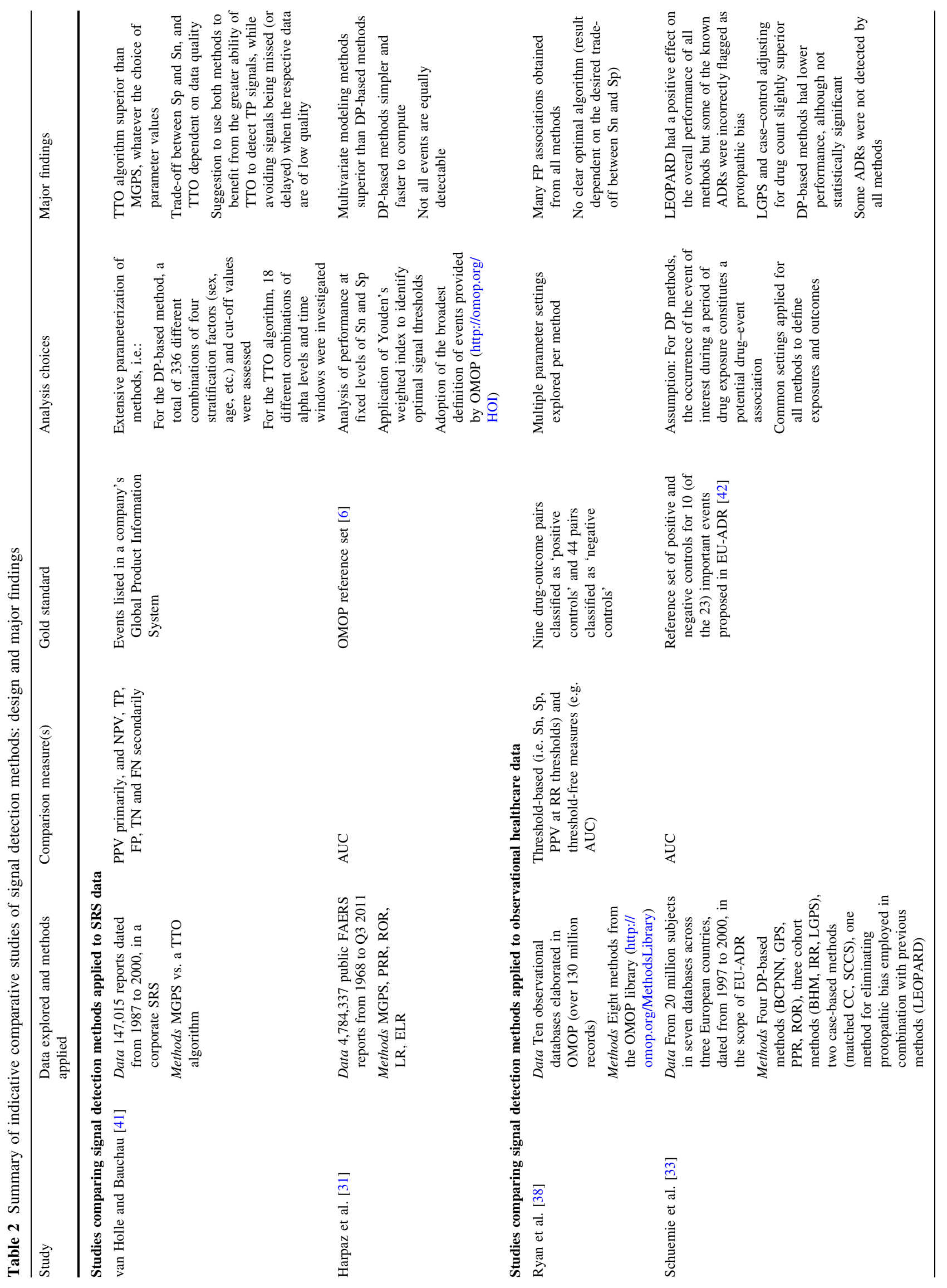




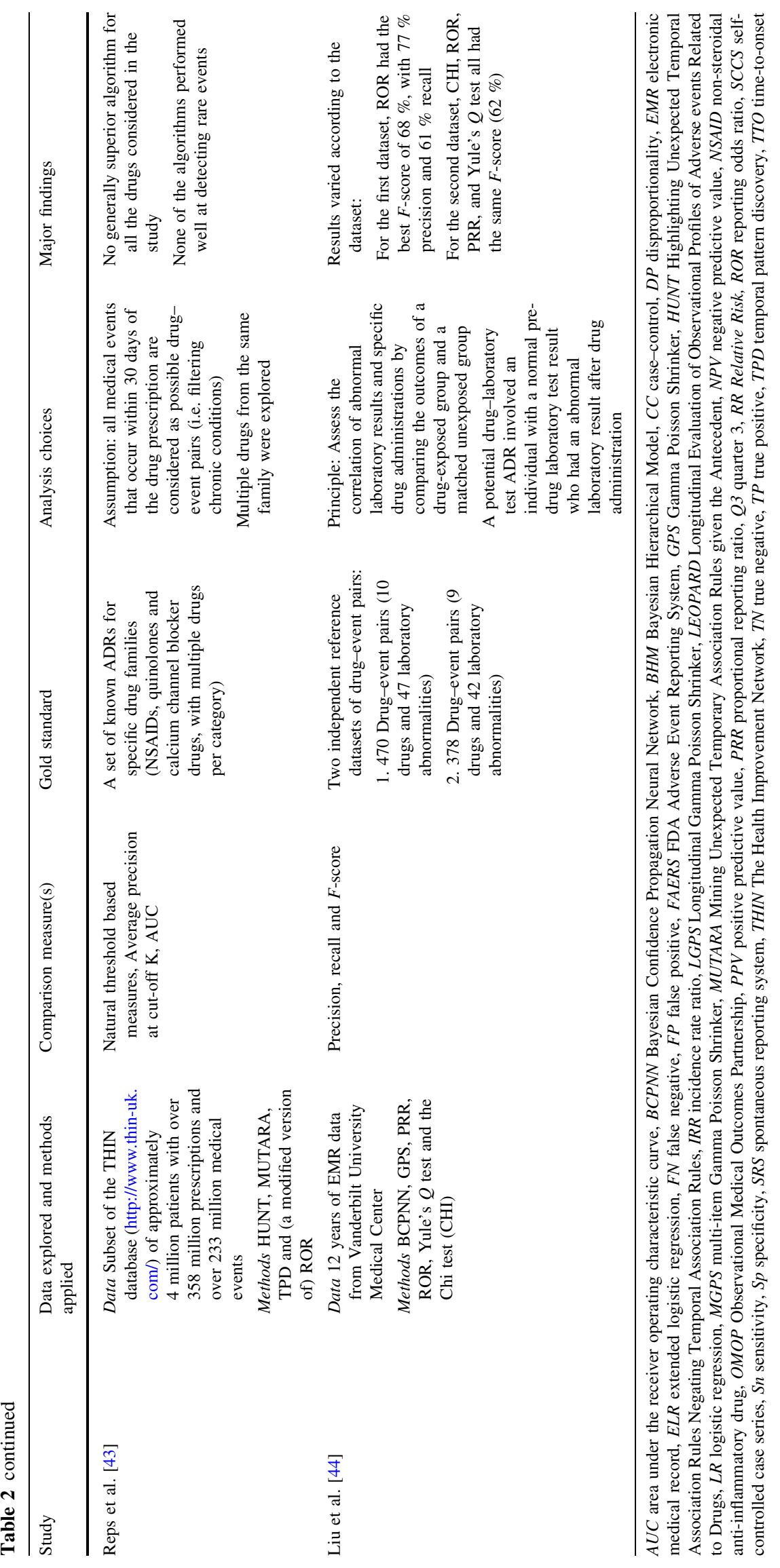


analysis of 4 million reports obtained from the FDA Adverse Event Reporting System (FAERS) and information extracted from 1.2 million EHR narratives, using DP analysis, in order to generate a highly selective ranked set of candidate ADRs and, consequently, advance the accuracy of signal detection. Ranking of outcomes relied on the 'Precision at K' metric [46]. The focus was on three serious adverse reactions, while a reference set of over 600 established and plausible ADRs was used to evaluate the proposed approach against the single, FAERS-based signal detector.

The combined signaling system demonstrated a statistically significant large improvement over the FAERS in the precision of top-ranked signals (i.e. from $31 \%$ to almost threefold for different evaluation categories). Probably even more important, this combinatorial analysis enabled the detection of a new association between a drug agent and an event that was supported by clinical review. Thus, the study concluded with promising initial evidence that exploring FAERS and EHR data in the scope of replicated signaling can improve the accuracy of signal detection in specific cases.

\subsection{Signal Detection by Integrating Chemical, Biological and Phenotypic Properties of Drugs}

The study of Liu et al. [47] followed an integrative perspective for ADR prediction by employing data beyond the phenotype level. Specifically, ADRs were predicted by jointly exploiting chemical (e.g. compound fingerprints or substructures), biological (including protein targets and pathways), and phenotypic properties of drugs (including indications and other known ADRs). Interestingly, the study suggested an approach for ADR prediction by combining the above types of information at the different stages of drug surveillance, i.e. chemical and biological for preclinical drug screening, and chemical, biological and phenotypic for postmarket surveillance.

This integrative analysis was focused on the prediction of 1385 known ADRs of 832 approved drugs, through five different analysis methods, namely logistic regression, naïve Bayes, K-nearest neighbor, random forest and a support vector machine. The elaborated data were obtained from public databases, while the evaluation was based on accuracy, precision, and recall, which were obtained from the best operating points of the global receiver operating characteristic (ROC) curve (resulting from merging the prediction scores for all ADRs). The study indicated that from the three types of information, phenotypic data were the most informative for ADR prediction. However, when biological and phenotypic features were added to the baseline chemical information, the proposed prediction model achieved significant improvements and successfully predicted ADRs associated with the withdrawal of specific drugs.

\section{Towards Semantically-Enriched, Integrated Signal Detection}

\subsection{Rationale}

The studies presented in Sect. 3 differ, not only regarding the employed data but also in the computational methods used. Nevertheless, both studies explored ways to reinforce signal detection outcomes either via replicated signaling or by integrating phenotype data with biological and chemical drug information, respectively, with significant findings. Given the heterogeneity of data sources and the variety of computational methods employed for signal detection, in order to further elaborate and systematically establish such combinatorial approaches we find the adoption of methods and tools originating from the field of semantic technologies and knowledge engineering important.

As illustrated in Fig. 1, combinatorial signal detection scales up from (a) studies where one data source is being explored by a single computational method (in a kind of 'coupled' setting); (b) benchmarking studies (in which one data source is being explored by various methods to enable comparisons in the methods' performance); and (c) studies focusing on replication of outcomes (i.e. one method applied to various data sources, typically of the same type), to settings where multiple data sources are explored by diverse methods. This may be seen as a natural evolution thanks to the increase in data availability for analysis, the development of more efficient computational methods, and the need for more accurate and evidence-based assessments.

Going a step further, Fig. 2 provides an overview of the signal detection process within an integrated perspective, where we discriminate among the diverse data sources considered for signal detection (part A), the respective computational signal detection methods per data source type (part B), the overall signal detection workflow that has to be supported in this integrated setting (part C), and the stakeholders involved in signal detection (part D), who use or contribute the above data sources and methods under a common framework. Ideally, the computational signal detection workflow in this case would first require mapping the analysis requirements defined by stakeholders (step 1) to the appropriate datasets and computational methods, and then (upon selection) launching the analysis (step 2). Next, aggregation of the output from the involved computational methods shall be performed (step 3), and then subsequently evaluating and ranking the provided indications based on reference knowledge sources, before providing the outcomes of the analysis to the respective end-users (step 4). 


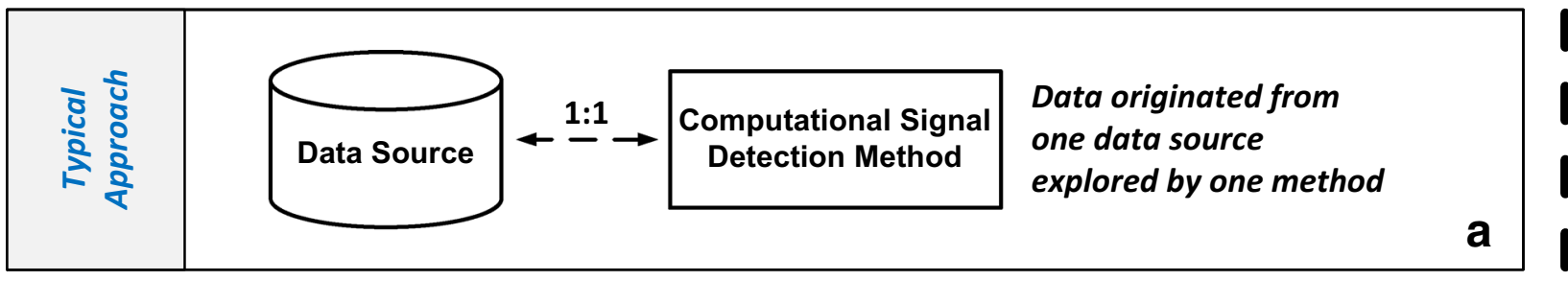

$\frac{1}{5}$
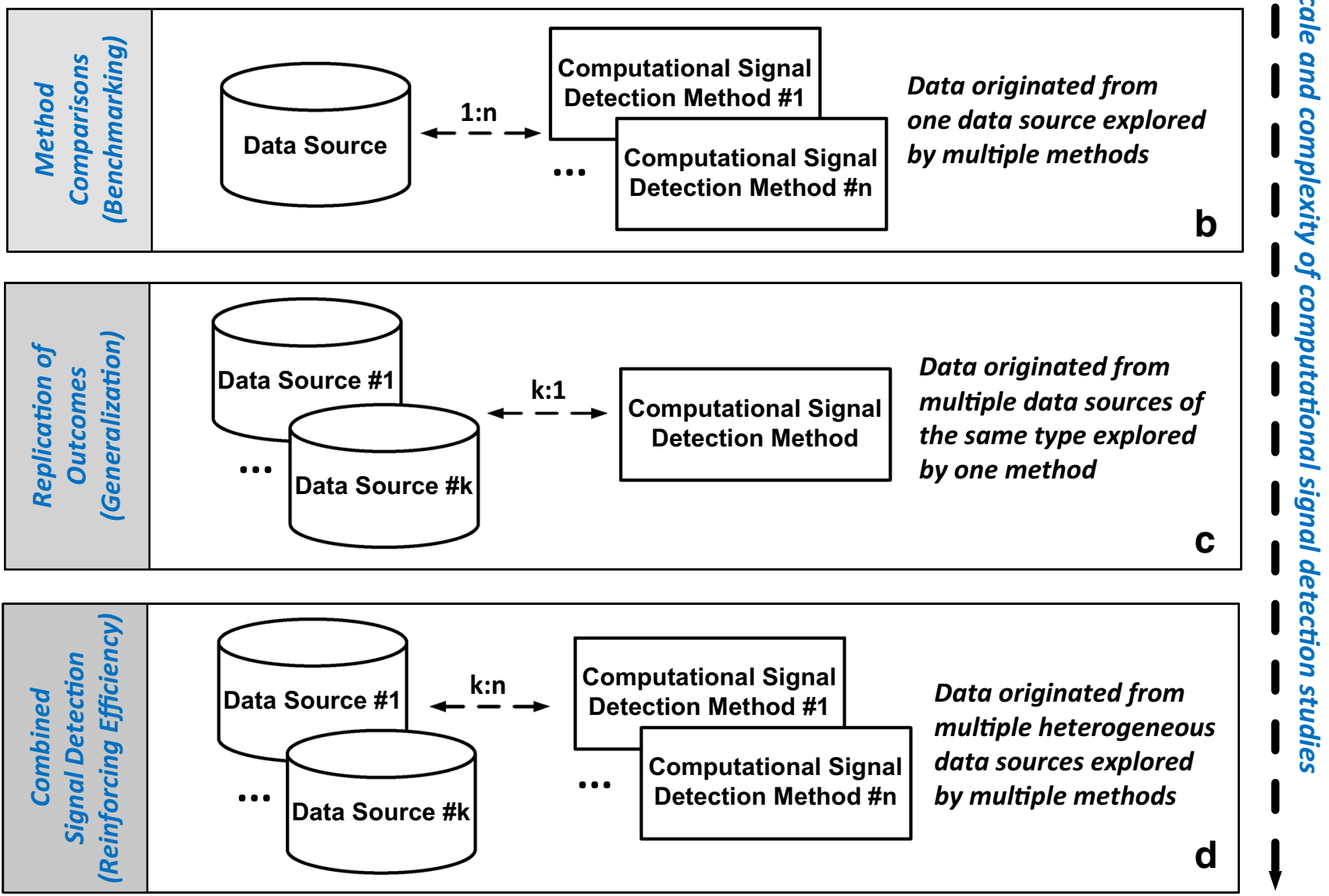

Fig. 1 Scaling-up computational signal detection towards combinatorial-integrated approaches: a the quite typical approach of one data source being explored by a single method in a 'coupled' fashion; $\mathbf{b}$ the benchmarking setting, i.e. one data source explored by various methods to enable the methods' comparison; c studies assessing replication of outcomes, i.e. one method applied to various data sources (typically of the same type); and $\mathbf{d}$ the integrated perspective, i.e. various data sources of different types explored by diverse methods in parallel

\subsection{Challenges for Large-Scale, Combinatorial Signal Detection}

The above scenario foresees the provision of uniformcombined access to data and computational methods for the end-users by hiding the underlying technical complexities. To this end, it is evident that the combinatorial signal detection approach poses new challenges for its realization, especially within a large-scale setting involving heterogeneous data sources and different methods, as well as the potential collaboration among stakeholders. Beyond the need to cope with privacy issues related to health data integration from different sources [48], big data analytics
[49], and hybrid signaling strategies [41], we discriminate the following issues (I represents 'issue'):

I.1 Employing an appropriate description schema to express quality attributes of data sources, useful for signal detection, as regards their structure, content and provenance. For SRSs, example attributes may be the coverage of predominant drugs and adverse effects, as well as the level of seriousness of the reports. Attributes for observational data sources may be the population size and the observation period. Similarly, a concise description of signal detection methods with respect to their input, output and analysis parameters 


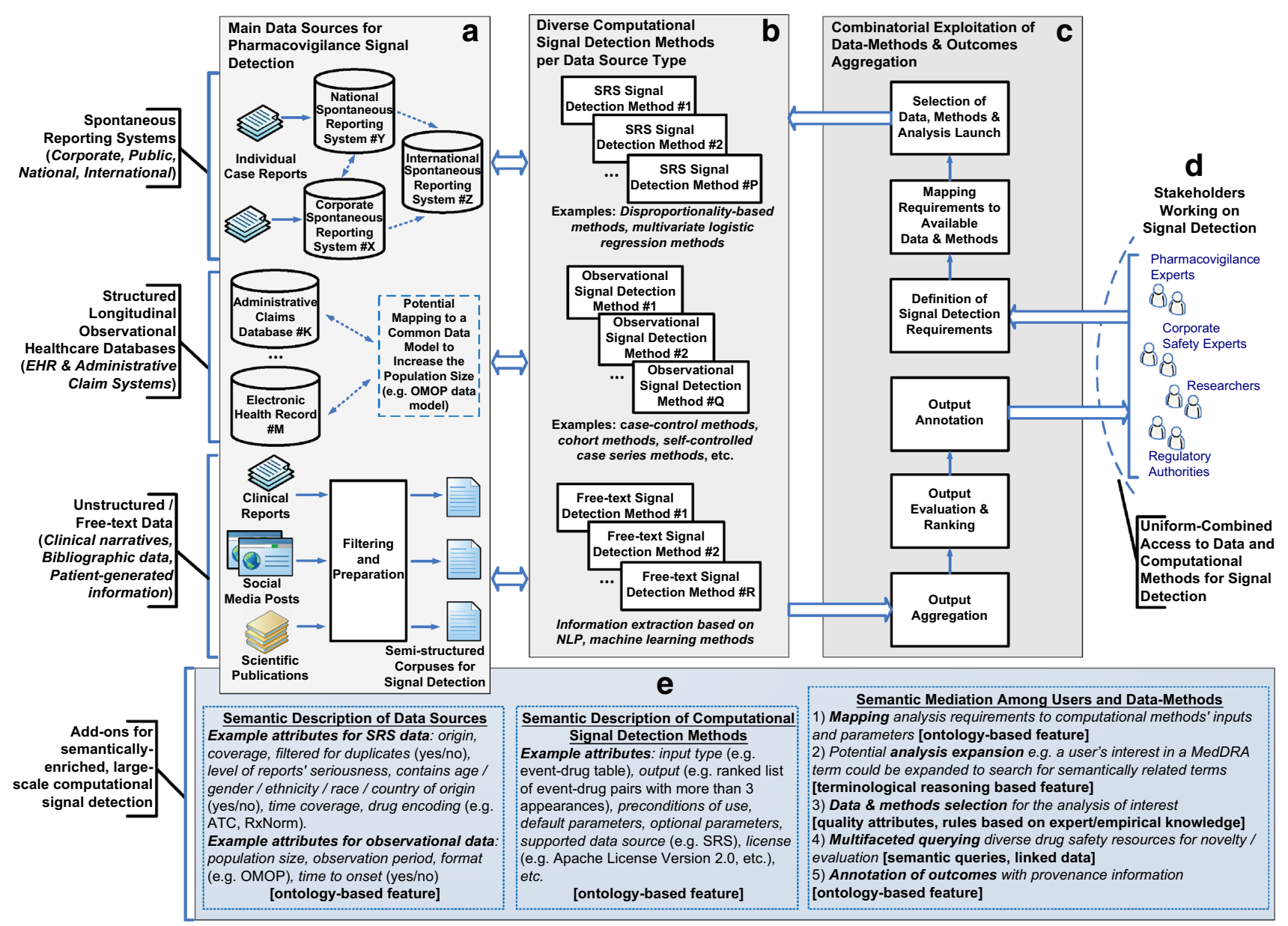

Fig. 2 Overview of the computational signal detection process in an integrated perspective. a Diverse data sources; b relevant computational signal detection methods per data source type; $\mathbf{c}$ the signal detection workflow; $\mathbf{d}$ stakeholders involved in signal detection for whom uniformcombined access to data and computational methods for signal detection shall be provided; e proposed add-ons for semantically-enriched, largescale signal detection. ATC Anatomical Therapeutic Chemical classification system, EHR electronic health record, MedDRA Medical Dictionary for Regulatory Activities, NLP Natural Language Processing, OMOP Observational Medical Outcomes Partnership, SRS spontaneous reporting system

is required, enabling their combined use either in parallel or in a conditional pipeline based on the output of individual steps. These descriptions shall be used by stakeholders contributing data and methods to the framework, in order to facilitate their selection and combined use by stakeholders interested in conducting signal detection.

I.2 The concrete definition of study parameters, concerning, for example, the drugs and health outcomes of interest (HOIs), enabling consistent comparisons of analysis experiments and their findings. Studies exploring observational data illustrated that this issue is of paramount importance and has a major impact on the outcomes of the analysis $[35,50]$.

I.3 Hiding the technical complexity in using signal detection method implementations and guide users to select and fine-tune their parameters. A significant variation among researchers in study design choices for signal detection has been highlighted by Stang et al. [51], due to various factors. Thus, it is important to provide guidance on study setup, given the focus of a signal analysis experiment. The PROMPT tool of Mini-Sentinel constitutes relevant work along this line (http://mini-sentinel.org/methods/methods_development/details.aspx?ID=1044).

I.4 Supporting evaluation of analysis outcomes in comparison with reference sources for both novelty detection (i.e. to exclude known ADRs) and acquisition of supportive evidence (e.g. biological attributes of drugs).

I.5 The common description of analysis results originated from experiments involving multiple data sources and signal detection method implementations along with the parameterization applied, including provenance 
information (i.e. the origin of the results with respect to the data, methods and parameter values that have been employed). Such a description will facilitate results sharing, systematic comparisons and assessing whether replication of experiments leads to similar results.

\subsection{Semantically-Enriched Integrated Signal Detection}

In order to address the above issues, it is imperative to establish systematic frameworks capable of supporting the concurrent exploitation of diverse data and methods. Semantic technologies bring many tools and benefits to cope with these aspects [52]. Examples of such technologies include ontologies (i.e. formal descriptions of the meaning of concepts), mappings among concepts/terms used in heterogeneous systems with the same meaning (semantic mapping), query languages, knowledge representation formalisms such as rules, as well as automatic inference mechanisms. To this end, in Fig. 2 we introduce an add-on layer (part E) with specific components relying on semantic technologies to conduct semantically-enriched, integrated signal detection comprising of the following elements $(E$ represents 'element'):

E.1 Ontologies for annotating with quality attributes datasets and methods involved in signal detection (to address issue I.1). To some extent, such an annotation has been elaborated in the scope of defining common data models to facilitate the analysis of observational data from various sources [34], with a major focus on the syntactic level, rather than on semantics. Moreover, a common description of methods is missing.

E.2 Semantic mappings between terminologies and/or ontologies obtained from ontology repositories (e.g. BioPortal, http://bioportal.bioontology.org/) that can facilitate the consistent definition of health outcomes and/or drugs of interest for a given signal detection scenario exploring diverse data sources through terminological/ontological reasoning (to address issue I.2).

E.3 Semantic rules linking the above quality attributes in order to support users in selecting the appropriate data, analysis methods and parameter settings for their drug surveillance use case, e.g. the targeted $\operatorname{drug}(\mathrm{s})$ or health conditions of interest (to address issue I.3).

E.4 Multifaceted querying of diverse drug safety resources for novelty assessment and for obtaining supporting evidence to interpret the findings of signal detection (to address issue I.4). This feature is possible thanks to the public availability of exploitable repositories of linked data in the domain of life sciences, such as Bio2RDF [53] and the EBI RDF platform [54], which provide programmatic access to resources such as chEMBL (https://www.ebi.ac.uk/chembl/), Clinical Trials.gov (http://clinicaltrials.gov/), DrugBank (http://www.drugbank.ca/) and SIDER (http:// sideeffects.embl.de/), to name a few.

E.5 Ontology-based annotation of outcomes with provenance information, enabling their sharing and facilitating comparisons (to address issue I.6).

We implement some of the above elements in the scope of the SAFER project (http://safer-project.eu/), which develops a semantically-enriched platform for combinatorial signal detection by exploring diverse opensource signal detection method implementations and publicly available data. In particular, we elaborate on the semantic harmonization of computational signal detection methods through an ontology model [55], aspiring to build a semantic registry of such methods and an integrated platform for experimentation on pharmacovigilance signal detection. Based on this ontology, we design and implement software interfaces to mediate between existing signal detection method implementations and to aggregate their outcomes, facilitating their exploitation under a common integrated framework. Besides using the built-in criteria of the employed computational methods for signal generation and ranking, we investigate other ways to address prioritization and management of the obtained outcomes (e.g. factors used in triage models [56, 57]), a challenging issue stemming from the parallel use of multiple signal detection methods that has also been remarked on by van Holle and Bauchau [41] and Harpaz et al. [45].

Notably, the Observational Health Data Sciences and Informatics (OHDSI, http://ohdsi.org/) collaborative elaborates on developing a global knowledge base (KB) bringing together and standardizing information for drugs and HOIs from various electronic sources relevant to drug safety [58]. This KB will be extremely useful for establishing a common reference for assessing the outcomes of computational signal detection methods, and is closely related to the element E.4 described above.

Taking into account the availability of diverse signal detection method implementations as open source (e.g. http://omop.org/MethodsLibrary, http://cran.r-project.org/ web/packages/PhViD/, http://mini-sentinel.org/methods/ methods_development/), public repositories of linked data related to drug safety, as well as initiatives for openly exposing drug safety data through programming interfaces such as openFDA (https://open.fda.gov/), we believe that, although challenging, the establishment of semantically- 
enriched platforms for integrated signal detection is feasible.

Although not explicitly related to the integrated signal detection perspective presented in this paper, semantic technologies have been employed in several drug safety applications, e.g. in order to automate signal detection, address heterogeneous data integration for safety assessment, normalize drug safety data, facilitate adverse event reporting, and semantically analyze case reports. Aiming to illustrate the applicability and added value of these technologies in the domain of drug safety, the following section refers to some interesting examples.

\section{Applications of Semantic Technologies in Drug Safety}

Bousquet et al. [59] developed PharmaMiner, a tool aiming to reinforce automated signal detection when using the Medical Dictionary for Regulatory Activities (MedDRA ${ }^{\circledR}$ ) to express adverse events. The study relied on the argument that automatic grouping of MedDRA ${ }^{\circledR}$ terms expressing similar medical conditions would increase the power of detection algorithms. Through an ontology, PharmaMiner employed terminological reasoning in order to group semantically-linked adverse events, and applied standard statistical analysis methods for the detection of potential signals. Evaluation of PharmaMiner with a dataset of 42,284 case reports extracted from the French Pharmacovigilance database illustrated that the approach enabled the identification of more occurrences of drug-ADR associations than using the original MedDRA ${ }^{\circledR}$ hierarchy, and could thus help pharmacovigilance experts to increase the number of responses to their queries when investigating case reports coded with MedDRA ${ }^{\circledR}$. Recently, Bousquet et al. extended the approach for automatically grouping MedDRA $^{\circledR}$ terms through the OntoADR ontology [60].

Stephens et al. [61] presented a use case of semantic web technologies for drug safety by focusing on heterogeneous data integration and analysis. The motivation was that semantic technologies can simplify data integration across multiple sources and support the logic to infer additional insights from the data. Given that effective decision making regarding a drug's safety profile requires the assessment of all the available information regarding, for example, the compound, the target and the patient group, Stephens et al. employed ontology-based inferencing and rules in order to guide decisions on either continuing to pursue a compound or withdrawing a drug from the market through the analysis of diverse data. The approach was illustrated via an example rule assessing the drug toxicity risk, which employed diverse parameters used along the drug discovery and development pipeline, such as the structural similarity of the compound of interest with others that failed due to toxicity, the compound binding to the target expressed with a number of single nucleotide polymorphisms (associated with the range of response to the drug), the therapeutic index, clinical findings, therapeutic dose, etc.

Wang et al. [62] elaborated on the normalization of FAERS data through semantic technologies. The objectives of their work were, first, improving the mining capacity of FAERS data for signal detection and, second, promoting semantic interoperability between the FAERS and other data sources. As drugs are registered in the FAERS by arbitrary names, e.g. trade names and abbreviations, and may even contain typographical errors, the lack of drug normalization introduces substantial barriers for data integration in signal detection. Wang et al. normalized drug information contained in the FAERS using RxNorm (http:// www.nlm.nih.gov/research/umls/rxnorm/), a standard terminology for medication, while drug class information was obtained from the US National Drug File Reference Terminology (NDF-RT, http://www.nlm.nih.gov/research/ umls/sourcereleasedocs/current/NDFRT). Regarding adverse event names, although the FAERS provides normalized terms based on MedDRA ${ }^{\circledR}$ preferred terms (PT), Wang et al. demonstrated that this normalization reinforces the data aggregation capability when linking PT terms to their corresponding system organ class (SOC) categories. The study resulted in a publicly available knowledge resource (http://informatics.mayo.edu/adepedia/index.php/ Download), which can be extended by connecting data from clinical notes, scientific literature, gene expression, etc., and various other ontologies.

Aiming to address interoperability issues that hamper the conduction of postmarketing safety analysis studies on top of EHR systems, the SALUS project (http://www. salusproject.eu/) built a semantic framework and a dedicated toolkit to serve this purpose. An interesting part of this toolkit was a component aimed at facilitating spontaneous reporting by prepopulating the respective forms with relevant EHR data [63]. To this end, standard (e.g. based on HL7) and proprietary EHR data models were mapped to the E2B data model (Electronic Standards for the Transfer of Regulatory Information, http://estri.ich.org/), while terminology mapping and reasoning services were designed to ensure the automatic conversion of local EHR terminologies, e.g. International Classification of Diseases, Tenth Revision (ICD-10, http://www.who.int/ classifications/icd/en/) or Logical Observation Identifiers Names and Codes (LOINC ${ }^{\circledR}$, http://loinc.org/), to MedDRA $^{\circledR}$, which is dominant for adverse event reporting. The partial automation of the adverse event reporting process achieved through this tool is expected to contribute to the reduction of underreporting, which has been often argued as a limitation of SRSs. 
Sarntivijai et al. [64] employed an ontology to conduct a comparative analysis of adverse events associated with two different types of seasonal influenza vaccines, namely killed and live influenza vaccines. The study analyzed reports from the US Vaccine Adverse Event Reporting System (VAERS), referring to a number of vaccines of the above categories based on measures such as the proportional reporting ratio and Chi-square. The identified adverse events were grouped using the Ontology of Adverse Events (OAE, http://www.oae-ontology.org/), based on their semantic similarity. This approach provided better classification results compared with MedDRA ${ }^{\circledR}$ and Systematized Nomenclature of Medicine-Clinical Terms (SNOMED-CT ${ }^{\circledR}$ )-based classifications. The analysis indicated that live influenza vaccines had a lower chance of inducing two severe adverse events compared with the other vaccine category, while previously reported positive correlation between one of the serious events and influenza vaccine immunization was based on trivalent influenza vaccines rather than monovalent influenza vaccines.

\section{Discussion and Conclusions}

Lately, signal detection has been a very active research field, demonstrating not only important advances but also many new challenges $[4,28,65,66]$. Further data sources are considered for signal detection and, consequently, new computational methods and approaches are constantly being proposed for their analysis [16, 26, 27]. Important findings as regards the capacity of new/established methods and data sources for signal detection have been illustrated by a number of comparative studies [31, 33, 38, 41, 43, 44]. Although it is arguable to what extent the outcomes of these studies are generalizable, it is clear that the concurrent use of multiple methods and data sources is essential.

Interestingly, increased drug surveillance through the synthesis of all possible information sources has been suggested by regulatory bodies [67] and highlighted in the literature [68]. Given the need to obtain more reliable and timely insights on drug safety risks, it has been reasonably argued that combining information across data sources could lead to more effective and accurate signal detection. These combinatorial investigations are expected to increase evidence on the obtained results, or provide new insights that may be not possible by investigating a single source. Some early successful examples of this approach for signal detection have been recently presented in the literature $[45,47]$.

However, in order to explore this perspective in its full potential we need systematic frameworks that will facilitate pharmacovigilance stakeholders to seamlessly share, access, and effectively use different data sources and computational methods for signal detection. In this article, we highlighted the challenges towards such a development and argued that semantic technologies bring the technical endeavor for this advancement. We also introduced concrete elements towards an integrated, semantically-enriched signal detection framework, spanning from the description of data sources and computational methods for selection, support in study setup, advanced access to reference linked-data resources for evaluation, and uniform description of the obtained outcomes.

The applicability and virtue of semantic technologies in drug safety have been illustrated in several applications. Aligned with the integrated signal detection perspective, we are currently developing a semantically-enriched signal detection platform relying on the semantic harmonization of signal detection methods and data sources [55]. Our research complements other efforts in the field, such as the OHDSI KB [63] and the SALUS semantic interoperability platform and tools [58], bringing a new perspective on large-scale, knowledge-intensive signal detection, and aspiring to increase efficiency, automation, support and collaboration for pharmacovigilance stakeholders.

Acknowledgments This research was supported by a Marie Curie Intra European Fellowship within the 7th European Community Framework Programme FP7/2007-2013 under Research Executive Agency (REA) Grant agreement No. 330422-the SAFER project. Vassilis Koutkias and Marie-Christine Jaulent have no conflicts of interest to declare.

Open Access This article is distributed under the terms of the Creative Commons Attribution Noncommercial License which permits any noncommercial use, distribution, and reproduction in any medium, provided the original author(s) and the source are credited.

\section{References}

1. World Health Organization. A practical handbook on the pharmacovigilance of antimalarial medicines. Geneva: WHO Document Production Services; 2008.

2. Council for International Organizations of Medical Sciences. Practical aspects of signal detection in pharmacovigilance: report of CIOMS Working Group VIII. Geneva: Council for International Organizations of Medical Sciences; 2010.

3. Hauben M, Madigan D, Gerrits CM, Walsh L, Van Puijenbroek EP. The role of data mining in pharmacovigilance. Expert Opin Drug Saf. 2005;4:929-48.

4. Harpaz R, DuMouchel W, Shah NH, Madigan D, Ryan P, Friedman C. Novel data-mining methodologies for adverse drug event discovery and analysis. Clin Pharmacol Ther. 2012;91:1010-21.

5. Reisinger SJ, Ryan PB, O'Hara DJ, et al. Development and evaluation of a common data model enabling active drug safety surveillance using disparate healthcare databases. J Am Med Inform Assoc. 2010;17:652-62. 
6. Ryan PB, Schuemie MJ, Welebob E, Duke J, Valentine S, Hartzema AG. Defining a reference set to support methodological research in drug safety. Drug Saf. 2013;36:S33-47.

7. Norén NG, Hopstadius J, Bate A, Star K, Edwards RI. Temporal pattern discovery in longitudinal electronic patient records. Data Min Knowl Discov. 2010;20:361-87.

8. Madigan D, Schuemie MJ, Ryan PB. Empirical performance of the case-control design: lessons for developing a risk identification and analysis system. Drug Saf. 2013;36:S73-82.

9. Ryan PB, Schuemie MJ, Madigan D. Empirical performance of the self-controlled cohort method: lessons for developing a risk identification and analysis system. Drug Saf. 2013;36:S95-106.

10. Suchard MA, Zorych I, Simpson SE, Schuemie MJ, Ryan PB, Madigan D. Empirical performance of the self-controlled case series design: lessons for developing a risk identification and analysis system. Drug Saf. 2013;36:S83-93.

11. Ryan PB, Schuemie MJ, Gruber S, Zorych I, Madigan D. Empirical performance of a new user cohort method: lessons for developing a risk identification and analysis system. Drug Saf. 2013;36:S59-72.

12. DuMouchel B, Ryan PB, Schuemie MJ, Madigan D. Evaluation of disproportionality safety signaling applied to healthcare databases. Drug Saf. 2013;36:S123-32.

13. Hauben M, Norén GN. A decade of data mining and still counting. Drug Saf. 2010;36:527-34.

14. van Puijenbroek EP, Bate A, Leufkens HG, Lindquist M, Orre R, Egberts AC. A comparison of measures of disproportionality for signal detection in spontaneous reporting systems for adverse drug reactions. Pharmacoepidemiol Drug Saf. 2002;11:3-10.

15. Hauben M, Bate A. Decision support methods for the detection of adverse events in post-marketing data. Drug Discov Today. 2009; 14:343-57.

16. Caster O, Juhlin K, Watson S, Norén GN. Improved statistical signal detection in pharmacovigilance by combining multiple strength-ofevidence aspects in vigiRank. Drug Saf. 2014;37:617-28.

17. Schuemie MJ. Methods for drug safety signal detection in longitudinal observational databases: LGPS and LEOPARD. Pharmacoepidemiol Drug Saf. 2011;20:292-9.

18. Zorych I, Madigan D, Ryan P, Bate A. Disproportionality methods for pharmacovigilance in longitudinal observational databases. Stat Methods Med Res. 2013;22:39-56.

19. Suling M, Pigeot I. Signal detection and monitoring based on longitudinal healthcare data. Pharmaceutics. 2012;4:607-40.

20. Friedman C, Elhadad N. Natural language processing in health care and biomedicine. In: Shortliffe EH, Cimino JJ, editors. Biomedical informatics. London: Springer; 2014. p. 255-84.

21. Chapman WW, Nadkarni PM, Hirschman L, D'Avolio LW, Savova GK, Uzuner O. Overcoming barriers to NLP for clinical text: the role of shared tasks and the need for additional creative solutions. J Am Med Inform Assoc. 2011;18:540-3.

22. Haerian K, Varn D, Vaidya S, Ena L, Chase HS, Friedman C. Detection of pharmacovigilance-related adverse events using electronic health records and automated methods. Clin Pharmacol Ther. 2012;92:28-34.

23. LePendu P, Iyer SV, Bauer-Mehren A, et al. Pharmacovigilance using clinical notes. Clin Pharmacol Ther. 2013;93:547-55.

24. Shetty KD, Dalal SR. Using information mining of the medical literature to improve drug safety. J Am Med Inform Assoc. 2011;18:668-74.

25. Shang N, Xu H, Rindflesch TC, Cohen T. Identifying plausible adverse drug reactions using knowledge extracted from the literature. J Biomed Inform. 2014;52:293-310.

26. Freifeld CC, Brownstein JS, Menone CM, et al. Digital drug safety surveillance: monitoring pharmaceutical products in Twitter. Drug Saf. 2014;37:343-50.
27. White RW, Harpaz R, Shah NH, DuMouchel W, Horvitz E. Toward enhanced pharmacovigilance using patient-generated data on the Internet. Clin Pharmacol Ther. 2014;96:239-46.

28. Harpaz R, Callahan A, Tamang S, et al. Text mining for adverse drug events: the promise, challenges, and state of the art. Drug Saf. 2014;37:777-90.

29. Sakaeda T, Tamon A, Kadoyama K, Okuno Y. Data mining of the public version of the FDA Adverse Event Reporting System. Int J Med Sci. 2013;10:796-803.

30. Tatonetti NP, Fernald GH, Altman RB. A novel signal detection algorithm for identifying hidden drug-drug interactions in adverse event reports. J Am Med Inform Assoc. 2012;19:79-85.

31. Harpaz R, DuMouchel W, LePendu P, Bauer-Mehren A, Ryan P, Shah NH. Performance of pharmacovigilance signal-detection algorithms for the FDA adverse event reporting system. Clin Pharmacol Ther. 2013;93:539-46.

32. Maignen F, Hauben M, Hung E, Holle LV, Dogne JM. A conceptual approach to the masking effect of measures of disproportionality. Pharmacoepidemiol Drug Saf. 2014;23:208-17.

33. Schuemie MJ, Coloma PM, Straatman H, et al. Using electronic health care records for drug safety signal detection: a comparative evaluation of statistical methods. Med Care. 2012;50:890-7.

34. Hartzema AG, Reich CG, Ryan PB, et al. Managing data quality for a drug safety surveillance system. Drug Saf. 2013;36:S49-58.

35. Reich CG, Ryan PB, Schuemie MJ. Alternative outcome definitions and their effect on the performance of methods for observational outcome studies. Drug Saf. 2013;36:S181-93.

36. Chazard E, Ficheur G, Bernonville S, Luyckx M, Beuscart R. Data mining to generate adverse drug events detection rules. IEEE Trans Inf Technol Biomed. 2011;15:823-30.

37. Li Y, Salmasian H, Vilar S, Chase H, Friedman C, Wei Y. A method for controlling complex confounding effects in the detection of adverse drug reactions using electronic health records. J Am Med Inform Assoc. 2014;21:308-14.

38. Ryan PB, Madigan D, Stang PE, Overhage JM, Racoosin JA, Hartzema AG. Empirical assessment of methods for risk identification in healthcare data: results from the experiments of the Observational Medical Outcomes Partnership. Stat Med. 2012;31:4401-15.

39. Norén GN. Pharmacovigilance for a revolving world: prospects of patient-generated data on the Internet. Drug Saf. 2014;37:761-4.

40. Banerjee AK, Okun S, Edwards IR, et al. Patient-reported outcome measures in safety event reporting: PROSPER Consortium guidance. Drug Saf. 2013;36:1129-49.

41. van Holle L, Bauchau V. Signal detection on spontaneous reports of adverse events following immunisation: a comparison of the performance of a disproportionality-based algorithm and a timeto-onset-based algorithm. Pharmacoepidemiol Drug Saf. 2014;23:178-85.

42. Trifirò G, Pariente A, Coloma PM, et al. Data mining on electronic health record databases for signal detection in pharmacovigilance: which events to monitor? Pharmacoepidemiol Drug Saf. 2009;18:1176-84.

43. Reps J, Garibaldi J, Aickelin U, Soria D, Gibson J, Hubbard R. Comparison of algorithms that detect drug side effects using electronic healthcare database. Soft Comput. 2013;17:2381-97.

44. Liu M, McPeek Hinz ER, Matheny ME, et al. Comparative analysis of pharmacovigilance methods in the detection of adverse drug reactions using electronic medical records. J Am Med Inform Assoc. 2013;20:420-6.

45. Harpaz R, Vilar S, Dumouchel W, et al. Combing signals from spontaneous reports and electronic health records for detection of adverse drug reactions. J Am Med Inform Assoc. 2013;20:413-9.

46. Manning CD, Raghavan P, Schütze $H$. Introduction to information retrieval. Cambridge: University Press; 2008. 
47. Liu M, Wu Y, Chen Y, et al. Large-scale prediction of adverse drug reactions using chemical, biological, and phenotypic properties of drugs. J Am Med Inform Assoc. 2012;19:e28-35.

48. Kim S, Sung MK, Chung YD. A framework to preserve the privacy of electronic health data streams. J Biomed Inform. 2014;50:95-106.

49. McCormick TH, Ferrell R, Karr AF, Ryan PB. Big data, big results: knowledge discovery in output from large-scale analytics. Stat Anal Data Min. 2014;7:404-12.

50. Hansen RA, Gray MD, Fox BI, Hollingsworth JC, Gao J, Zeng P. How well do various health outcome definitions identify appropriate cases in observational studies? Drug Saf. 2013;36:S27-32.

51. Stang PE, Ryan PB, Overhage JM, Schuemie MJ, Hartzema AG, Welebob E. Variation in choice of study design: findings from the Epidemiology Design Decision Inventory and Evaluation (EDDIE) survey. Drug Saf. 2013;36:S95-106.

52. Domingue J, Fensel D, Hendler JA. Handbook of semantic web technologies. Berlin: Springer; 2011.

53. Callahan A, Cruz-Toledo J, Dumontier M. Ontology-based querying with Bio2RDF's linked open data. J Biomed Semant. 2013;4(Suppl 1):S1.

54. Jupp S, Malone J, Bolleman J, et al. The EBI RDF platform: linked open data for the life sciences. Bioinformatics. 2014;30:1338-9.

55. Koutkias V, Jaulent MC. Leveraging post-marketing drug safety research through semantic technologies: the PharmacoVigilance Signal Detectors Ontology. In: Proceedings of the International Workshop on Semantic Web Applications and Tools for Life Sciences (SWAT4LS): 9-11 Dec 2014, Berlin.

56. Lindquist M. Use of triage strategies in the WHO signal-detection process. Drug Saf. 2007;30:635-7.

57. Strandell J, Caster O, Hopstadius J, Edwards IR, Norén GN. The development and evaluation of triage algorithms for early discovery of adverse drug interactions. Drug Saf. 2013;36:371-88.

58. Boyce RD, Ryan PB, Norén GN, et al. Bridging islands of information to establish an integrated knowledge base of drugs and health outcomes of interest. Drug Saf. 2014;37:557-67.
59. Bousquet C, Henegar C, Louët AL, Degoulet P, Jaulent MC. Implementation of automated signal generation in pharmacovigilance using a knowledge-based approach. Int J Med Inform. 2005;74:563-71.

60. Bousquet C, Sadou E, Souvignet J, Jaulent MC, Declerck G. Formalizing MedDRA to support semantic reasoning on adverse drug reaction terms. J Biomed Inform. 2014;49:282-91.

61. Stephens S, Morales A, Quinlan M. Applying semantic web technologies to drug safety determination. IEEE Intell Syst. 2006;21:82-6.

62. Wang L, Jiang G, Li D, Liu H. Standardizing adverse drug event reporting data. J Biomed Semant. 2014;5:36.

63. Declerck G, Hussain S, Daniel C, et al. Bridging data models and terminologies to support adverse drug event reporting using EHR data. Methods Inf Med. 2014;54:24-31.

64. Sarntivijai S, Xiang Z, Shedden KA, et al. Ontology-based combinatorial comparative analysis of adverse events associated with killed and live influenza vaccines. PLoS One. 2012;7(11):e49941.

65. Platt R, Carnahan R, editors. The US Food and Drug Administration's Mini-Sentinel Program. Pharmacoepidemiol Drug Saf. 2012;21:S1-303. http://onlinelibrary.wiley.com/doi/10.1002/pds. v21.S1/issuetoc

66. Evans SJW, editor. Studying the science of observational research: empirical findings from the Observational Medical Outcomes Partnership. Drug Saf. 2013;36:S1-204. http://link. springer.com/journal/40264/36/1/suppl/page/1

67. European Commission. eHealth for safety: impact of ICT on patient safety and risk management. 2007. Available at: http:// www.ehealth-for-safety.org/news/documents/eHealth-safetyreport-final.pdf. Accessed 12 Feb 2015.

68. Kelman CW, Pearson SA, Day RO, Holman CD, Kliewer EV, Henry DA. Evaluating medicines: let's use all the evidence. Med J Aust. 2007;186:249-52. 